\title{
Methods for discovering genomic loci exhibiting complex patterns of differential methylation
}

\author{
Thomas J. Hardcastle (D)
}

\begin{abstract}
Background: Cytosine methylation is widespread in most eukaryotic genomes and is known to play a substantial role in various regulatory pathways. Unmethylated cytosines may be converted to uracil through the addition of sodium bisulphite, allowing genome-wide quantification of cytosine methylation via high-throughput sequencing. The data thus acquired allows the discovery of methylation 'loci'; contiguous regions of methylation consistently methylated across biological replicates. The mapping of these loci allows for associations with other genomic factors to be identified, and for analyses of differential methylation to take place.
\end{abstract}

Results: The segment Seq $\mathbf{R}$ package is extended to identify methylation loci from high-throughput sequencing data from multiple experimental conditions. A statistical model is then developed that accounts for biological replication and variable rates of non-conversion of cytosines in each sample to compute posterior likelihoods of methylation at each locus within an empirical Bayesian framework. The same model is used as a basis for analysis of differential methylation between multiple experimental conditions with the bayseq $\mathbf{R}$ package. We demonstrate the capability of this method to analyse complex data sets in an analysis of data derived from multiple Dicer-like mutants in Arabidopsis. This reveals several novel behaviours at distinct sets of loci in response to loss of one or more of the Dicerlike proteins that indicate an antagonistic relationship between the Dicer-like proteins at at least some methylation loci. Finally, we show in simulation studies that this approach can be significantly more powerful in the detection of differential methylation than many existing methods in data derived from both mammalian and plant systems.

Conclusions: The methods developed here make it possible to analyse high-throughput sequencing of the methylome of any given organism under a diverse set of experimental conditions. The methods are able to identify methylation loci and evaluate the likelihood that a region is truly methylated under any given experimental condition, allowing for downstream analyses that characterise differences between methylated and non-methylated regions of the genome. Futhermore, diverse patterns of differential methylation may also be characterised from these data.

Keywords: Methylation, DMRs, High-throughput sequencing, Epigenomics, Dicer

\section{Background}

Cytosine methylation, found in most eukaryotes and playing a key role in gene regulation and epigenetic effects [1-3], can be investigated at a genome wide level through high-throughput sequencing of bisulphite treated DNA [4]. Treatment of denatured DNA with sodium bisulphite deaminates unmethylated cytosines into uracil; sequencing this treated DNA thus allows, in principle, not only

Correspondence: tjh48@cam.ac.uk

Department of Plant Sciences, University of Cambridge, Downing Street,

CB2 3EA Cambridge, UK the identification of every methylated cytosine but an assessment of the proportion of cells in which the cytosine is methylated. Moreover, by comparing these quantitative methylation data across experimental conditions, genomic regions displaying differential methylation can be detected.

The data available for methylation locus finding from bisulphite treated DNA are generated from a set of sequencing libraries. Each library consists of a set of sequenced reads which can be aligned and summarised [5] to report at each cytosine the number of sequenced reads in which the cytosine is methylated, and the number 
in which the cytosine is unmethylated [6]. Several methods have been proposed to detect differential methylation at the cytosine level, and to identify contiguous differentially methylated cytosines defined as differentially methylated regions (DMRs) [7]. However, these approaches, in not identifying non-differentially methylated regions and unmethylated regions, preclude many strategies for downstream identification of the biological significance of differential methylation.

We propose here a new method for methylation analysis based on the notion of methylation 'loci'; genomic regions defined by the presence of contiguous cytosines whose methylation is correlated across experimental conditions (Fig. 1). Cytosines within a given locus may thus be assumed to share biogenesis and functional properties [8]. Furthermore, the identification of methylation 'loci' and the quantification of methylation within a locus increases statistical power to detect differential methylation. We show that by taking a novel approach in which methylation loci are identified and subsequently analysed for patterns of differential behaviour, we are able to achieve high levels of accuracy in identifying differential methylation. The empirical Bayesian methods employed also allow analysis of multiple patterns of differential methylation to be identified within complex data sets, allowing for detailed downstream analysis of biological mechanisms. We achieve this by adapting our previously described methods for defining small RNA (sRNA) loci from high-throughput sequencing of sRNAs [9], and for a generalised analysis of high-throughput sequencing data [10]. These methods allow for an analysis of differential behaviour in the methylome that accounts both for biological variation between replicates and systemic differences between samples caused by variations in the conversion rates of bisulphite treatment.

We demonstrate that this approach, in addition to allowing greater flexibility in analysis, offers better performance than a number of existing methods for the analysis of cytosine methylation data. We achieve this by simulating data using WGBSSuite [11], a recently developed stochastic method for generating simulated single base resolution DNA methylation data, with simulations based on parameters derived from both plant and mammalian systems. We demonstrate high performance under a variety

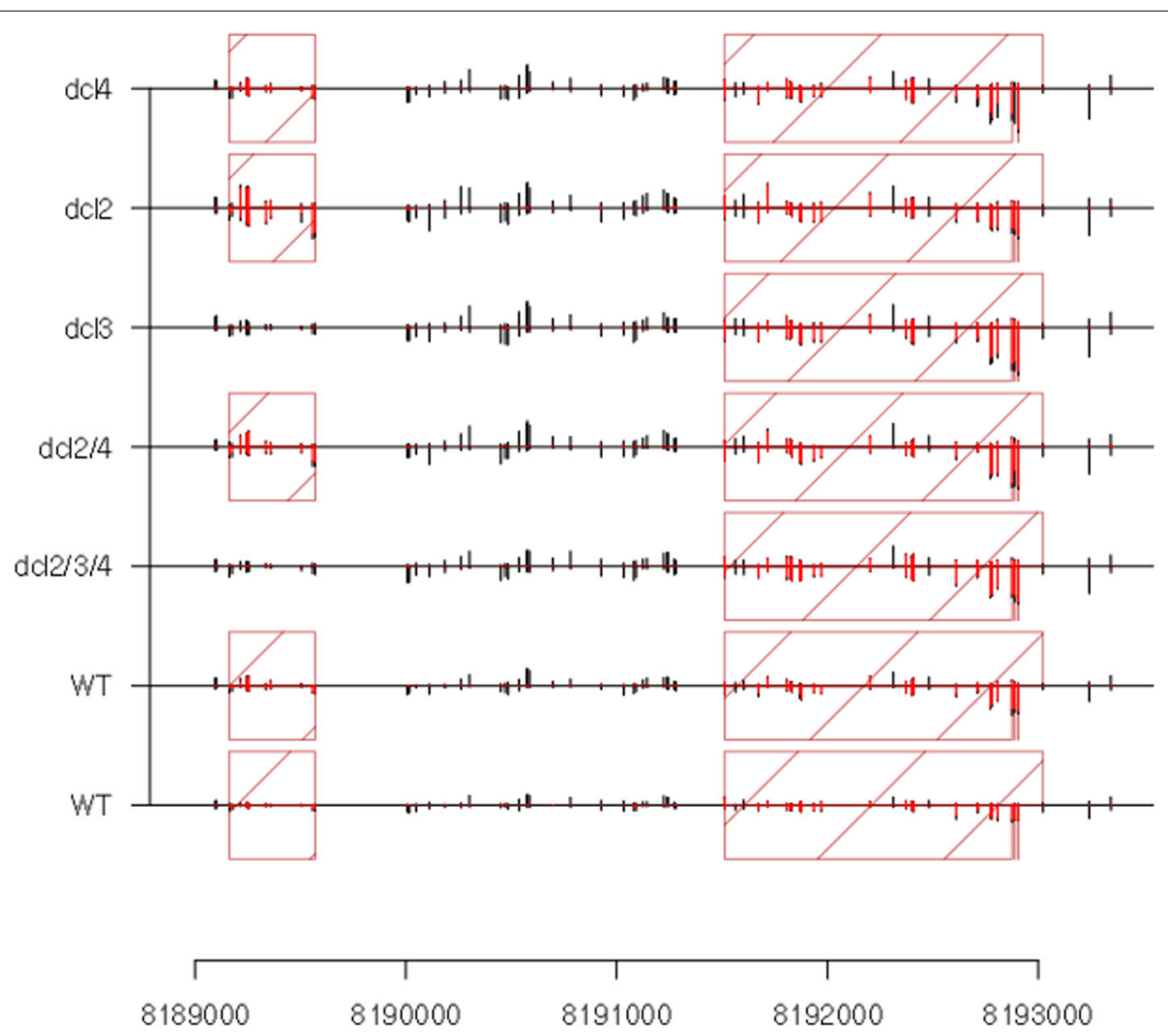

Fig. 1 Examples of methylation loci in a set of Arabidopsis thaliana mutants in the Dicer-like (dcl) proteins, and wild-type samples [12]. Each row represents a single biological sample; the height of the bars represents the number of sequenced reads in which unmethylated cytosines (black) and methylated cytosines (red) appear. Values above the horizontal lines represent cytosines on the positive strand while values below the horizontal lines represent cytosines on the negative strand. The dark red hatched boxes indicate the identified loci. On the left, a methylation locus is identified that is methylated in all samples except the $d c / 3$ and $d c / 2 / 3 / 4$ mutants, on the right, a locus that is methylated in all samples 
of simulation conditions, with substantial improvements over existing methods in the analysis of small changes in methylation between experimental conditions and for low numbers of biological replicates.

We further demonstrate these methods in an analysis of methylation in all contexts in mutants of the Dicer-like (DCL) proteins in Arabidopsis [12]. In higher plants, Dicer or Dicer-like proteins form a small gene family of sometimes overlapping function in the biogenesis of small RNAs [13]. In Arabidopsis, four different DCL proteins exist, acting in a partially redundant manner [14]. Predominantly, DCL1 is involved in the production of 21-nt miRNAs. DCL2 and DCL4 act redundantly and perhaps hierarchically to produce 22 and 21-nt sRNAs. DCL3 produces 24-nt sRNAs, previously identified as the key component of RNA-directed DNA methylation (RdDM). Recent work [15-17] has however emphasised the importance of 21 and 22-nt sRNAs, and hence of the DCL2 and DCL4 proteins, in regulating methylation at at least some loci. By applying the methods developed here we are able to identify multiple patterns of differential behaviour between the Dicer-like mutants and the loci which correspond to these.

\section{Methods}

\section{Candidate loci and nulls}

We analyse these data by adapting our previous methods for the identification of small RNA loci [9]. We begin by defining a set of candidate loci which may plausibly represent some methylation loci. A candidate locus begins and ends at some cytosine with a minimal proportion $p_{\text {min }}$ of methylation in at least one sequencing library. Considering all such loci is computationally infeasible and so filters are required to exclude implausible candidates and reduce the computational effort required. If two cytosines with a proportion of methylation above $p_{\text {min }}$ are within some minimal distance $d_{\min }$ they are assumed to lie within the same locus. We further restrict the set by removing from consideration any candidate locus containing a region greater than $\lambda_{\max }$ that contains no cytosine with a proportion of methylation above $p_{\text {min }}$. Candidate loci may be defined with respect to a single strand (by default), or combine the observed data from both strands. In the analyses presented here, we use $p_{\min }=0.05, d_{\text {min }}=2$, $\lambda_{\max }=1000$. These parameters have been found by experience to well characterise the methylation loci under most circumstances; however, the results identified will in most cases be robust to relatively large changes to these parameters.

We define the set of candidate nulls, regions which may represent a region without significant methylation, by considering the gaps between candidate loci. We refer to the regions separating each candidate locus from its nearest neighbour (in either direction) as 'empty'. Candidate nulls consist of the union of the set of 'empty' regions, the set of candidate loci extended into the empty region to their left, the set of candidate loci extended into the empty region to their right, and the set of candidate loci extended into the empty regions to both the left and right.

\section{Classification of candidate loci}

The data pertaining to the candidates defined above are the number of methylated and un-methylated cytosines sequenced and aligning to these regions for each sample. Biological replication is defined in terms of replicate groups, non-intersecting sets of biological replicates. Thus the samples may be thought of as the set $\left\{A_{1}, \cdots, A_{m}\right\}$ with a replicate structure defined by $\mathcal{R}=\left\{R_{1}, \cdots, R_{n}\right\}$ where $j \in R_{q}$ if and only if sample $A_{j}$ is a member of replicate group $q$. We then identify those candidates which represent at least part of a true locus of methylation, given the observed data for each replicate group.

For a replicate group $R_{q}$ and locus $i$ we consider the total number of methylated and unmethylated cytosines $u_{i q}=\sum_{j \in R_{q}} u_{i j}$ and $u_{i q}^{\prime}=\sum_{j \in R_{q}} u_{i j}^{\prime}$ respectively. For the purposes of identifying the methylation loci, we assume that these data are described by a binomial distribution with parameter $p_{i q}$ which has a beta prior distribution with parameters $(\alpha, \beta)$; we use an uninformative Jeffreys prior of $\alpha=\beta=\frac{1}{2}$. This assumption implicitly neglects biological variability between samples, which could be better modelled by a beta-binomial distribution. However, the computational cost involved in using a beta-binomial distribution is considerable. We therefore make this simplifying assumption in order to identify the loci but apply a model based on the beta-binomial distribution downstream to evaluate the likelihood that an identified locus represents a true methylation locus within a set of biological replicates.

The posterior distribution of the parameter $p_{i q}$ is then a beta distribution with parameters $\left(\alpha+u_{i q}, \beta+u_{i q}^{\prime}\right)$. A segment is identified as a methylation 'locus' if the posterior likelihood that $p_{i q}>t$ exceeds some critical value. Similarly, we can classify candidate nulls as true representatives of a null region by identifying those candidates with a posterior likelihood that $p_{i q}<t$ exceeding some critical value. By default, we use a required likelihood of $90 \%$. The parameter $t$ is intended to provide a threshold distinguishing regions of 'true' or biologically relevant methylation from low level background methylation attributable to biological or technical noise. The appropriate value for this parameter is contingent on many factors, including organism, methylation context, heterogeneity of sample and the assignment of biological meaning; we use a value of $20 \%$ here across all analyses with the caveat that this may be more or less appropriate to any individual experiment. 
The above analysis neglects the effect of non-conversion rates on the observed values for $u_{i q}$ and $u_{i q}^{\prime}$. The data observed for a given sample may be defined as the number of methylated $\left(C_{i j}\right)$ and unmethylated $\left(T_{i j}\right)$ sequenced cytosines at the $i$ th locus and $j$ th sample. However, if nonconversion of cytosines occurs, we might expect that the true number of methylated cytosines is somewhat lower, and the true number of unmethylated cytosines somewhat higher than the observed values.

We can find no closed form expression for the posterior if the effects of non-conversion rates on the distribution of the data are accounted for. However, we can adjust the observed data by the expected non-conversion rates by setting $u_{i j}=C_{i j}-\frac{Q_{j}}{1-Q_{j}} T_{i j}$ and $u_{i j}^{\prime}=T_{i j}+\frac{Q_{j}}{1-Q_{j}} T_{i j}$, where $Q_{j}$ is the non-conversion rate for sample $j$.

\section{Consensus loci}

Given a classification on the set of candidate loci and nulls, we identify a set of consensus loci given the classifications on sets of overlapping candidates in a similar manner to that described for sRNA loci [18]. We begin by assuming that a true locus of methylation should not contain a null region within a replicate group in which the locus is methylated. Thus, if some candidate locus $l_{i}$ is classified as a locus in a set of replicate groups $\Psi$, and there exists some candidate null $n_{j}$ that lies completely within $l_{i}$ and is classified as a null in one or more of the replicate groups in the set $\Psi$, we discard the locus $l_{i}$. Of the remaining candidate loci, we then rank those that remain by the number of replicate groups in which they are classified as a locus, settling ties by giving higher rank to the longest candidate loci. The consensus loci are then formed by choosing all those candidate loci that do not overlap with a higher ranked candidate, giving a non-overlapping set of loci on each strand. 'Null' loci are defined as the contiguous regions of the genome containing no identified locus.

\section{Likelihood of data}

We can compute posterior likelihoods of methylation and differential methylation on the identified loci through application of the empirical Bayesian methods described in Hardcastle (2016) [10]. Since the set of identified loci is considerably smaller than the set of all possible loci, we are able to incorporate biological variability into our models at this stage without the computational cost becoming excessive.

We achieve this by defining a distribution on the data accounting for biological variation between replicates. Ignoring issues of non-conversion, we would assume that the data in equivalently methylated samples are betabinomially distributed as in a straightforward analysis of paired data [18].

$$
\mathbb{P}\left(D_{i j} \mid p_{q}, \phi\right)=\left(\begin{array}{c}
C_{i j}+T_{i j} \\
C_{i j}
\end{array}\right) \frac{B\left(\alpha+C_{i j}, \beta+T_{i j}\right)}{B(\alpha, \beta)}
$$

Equation 1 defines the density function of the observed data $D_{i j}$, given a proportion of methylation $p_{q}$ for each equivalence class $E_{q}$ and a dispersion parameter $\phi$ capturing the level of variation between biological replicates. Then $\alpha=p_{q} \frac{1-\phi}{\phi}, \beta=\left(1-p_{q}\right) \frac{1-\phi}{\phi}$. Following our previous work [10], a joint distribution on $\left\{p_{q}, \phi\right\}$ may be empirically estimated by repeatedly sampling individual loci (without replacement) and estimating for each replicate group $q$ the values $\left\{p_{q}, \phi\right\}$ by maximum likelihood estimation based on the data observed at that locus. The dispersion parameter $\phi$ is assumed to be preserved across replicate groups and $p_{q}$ is not.

If non-conversion rates are estimable, we can first normalise the observed data as above and proceed assuming a beta-binomial distribution. However, this does not fully account for the stocasticity of non-conversion events at each cytosine. A full analysis incorporating nonconversion events requires that the data within each sample $j$ are assumed to be the sum of a binomial distribution with success parameter $Q_{j}$ (the rate of non-conversion) and a beta-binomial distribution with parameters $p_{q}$ (the expected proportion of methylated cytosines) and dispersion parameter $\phi$. Then the likelihood of the observed data $D_{i j}$ at a single locus $i$ for a sample $j$ is given by

$$
\begin{aligned}
\mathbb{P}\left(D_{i j} \mid Q_{j}, p, \phi\right)= & \sum_{m=0}^{C_{i j}}\left(\begin{array}{c}
T_{i j}+m \\
m
\end{array}\right) Q_{j}^{m}\left(1-Q_{j}\right)^{T_{i j} \times} \\
& \left(\begin{array}{c}
C_{i j}+T_{i j} \\
C_{i j}-m
\end{array}\right) \frac{B\left(\alpha+C_{i j}-m, \beta+T_{i j}+m\right)}{B(\alpha, \beta)}
\end{aligned}
$$

where $m$ is the number of unconverted unmethylated cytosines and the remaining parameters are as in Eq. 1. As before, we can then estimate an empirical distribution on the parameters $\left\{p_{q}, \phi\right\}$ may be empirically estimated by repeatedly sampling individual loci (without replacement) and fitting values for a sampled locus by maximum likelihood methods.

\section{Posterior likelihoods of methylation}

Given the empirically estimated joint distributions on the parameters of our distribution, we can estimate posterior likelihoods of methylation for each replicate group and locus using the methods described in Hardcastle (2016) [10]. We derive two empirical distributions on the parameters, one by sampling regions identified as methylation loci, and one by sampling regions identified as null regions.

Given these two distributions, we are able to calculate posterior likelihoods of methylation for each locus and replicate group by taking the product of the probability of the observed data at a locus under each distribution and an empirically determined prior on the likelihood of an arbitrary region being methylated or not in a given 
replicate group [10]. Regions exhibiting various patterns of differential methylation can be similarly identified using the density function defined in Eq. 2 or Eq. 1 (neglecting non-conversion rates) in the bayseq $\mathbf{R}$ package.

Since the definition of differential methylation is primarily concerned with a shift in ratios between the number of methylated cytosines and the number of unmethylated cytosines, it is possible for long regions of low methylation to exhibit patterns of differential methylation. We thus find improved performance by combining the likelihood of differential methylation within a locus with the likelihood of that locus being methylated in at least one replicate group. The final statistic used to identify DMRs with this method is thus:

$$
\mathbb{P}\left(M \mid D_{i j}\right)\left(1-\prod_{q}\left(1-\mathbb{P}\left(L_{q} \mid D_{E_{q}}\right)\right)\right)
$$

where $\mathbb{P}\left(M \mid D_{i j}\right)$ is the likelihood of a model $M$ of differential methylation given the observed data in each sample at the $i$ th defined region, and $\mathbb{P}\left(L_{i q} \mid D_{E_{q}}\right)$ is the likelihood that the $i$ th region defines an expressed locus within replicate group $q$.

\section{Results}

\section{Analysis of the methylome in $\mathrm{dcl}$ mutants of Arabidopsis}

We demonstrate the value of this approach in a reanalysis of the methylome in the Dicer-like mutants from the Stroud et al. (2013) [12] dataset. We identify in a single analysis methylation loci in the $d c l 2, d c l 3, d c l 4, d c l 2 / 4$ and $d c l 2 / 3 / 4$ mutants, together with wild-type samples and discover complex patterns of differential methylation that exist between these mutants and the wild-type samples.

We begin with a standard pipeline for read alignment and summarisation [5]. Reads were aligned and summarised for each methylation context using the Bismark caller [19] with default settings. Since cytosine methylation should be absent in the chloroplast and mitochondrial genomes, we can estimate non-conversion rates as the ratio of sequenced cytosines to thymines in the reads aligning to these genomes. This was done for each sample and incorporated into the analysis at the distributional level.

We separate the data into the three major contexts of methylation; $\mathrm{CpG}, \mathrm{CHG}$, and $\mathrm{CHH}$. For each context of methylation, we identify a set of loci and estimate posterior likelihoods that any given locus is truly methylated in each of the experimental conditions. Figure 2 summarises the input data and expected numbers of loci in each mutant, based on the posterior likelihoods. The genomewide trends in methylation remain relatively constant in all of the $d c l$ mutants relative to the wild-type samples, with some minor loss of methylation (relative to wildtype) at this scale in the $\mathrm{CHH}$ context in the $d c l 2 / 3 / 4$ and dcl3 mutants, and some gain of $\mathrm{CHH}$ methylation in $d c l 4$ mutant at the centromeric regions. The total number of methylation loci in each condition may be estimated by summing the posterior likelihoods of loci (Fig. 2d). Relative to wild-type, expected numbers of loci do not alter substantially for $d c l 2 / 4$ loci in any condition, or for CpG methylation in $d c l 2 / 3 / 4$, while all the single mutants show lower numbers of methylation in all contexts. The numbers of methylation loci discovered in the CHG context are substantially lower than for other contexts; however, the loci discovered are generally longer, as shown by the estimated portion of the genome covered by loci in each context (Fig. 2e), which shows roughly equivalent coverage for $\mathrm{CpG}$ and $\mathrm{CHG}$ with a minor reduction in $\mathrm{CHH}$ context.

We next consider patterns of differential methylation at the level of the identified loci. For each region of the genome, posterior likelihoods of difference are identified, and adjusted by the likelihood that the region is a methylation locus in at least one condition. From these posterior likelihoods, we can estimate the expected number of loci belonging to each model of equivalence and difference between the conditions as the sum of the posterior likelihoods for this model over all loci. We can also select specific loci by controlling the false discovery rate (FDR) estimated from the posterior likelihoods. Ten patterns of differential methylation (Fig. 3) are identified with an estimated number of loci greater than one thousand and a number of loci with an FDR $<0.05$ greater than two hundred in at least one methylation context. A fuller list of potential models and the numbers of loci corresponding to these is available in Additional file 1: Table S1.

The ten models selected for further consideration can be roughly partitioned into five classes based on their definitions and the contexts in which they are most commonly found. Model A represents those methylation loci which show no differential methylation. Unsurprisingly, these are common in all contexts of methylation, as the DCL-dependent methylation makes up a small proportion of the total methylation on the Arabidopsis genome. The next class is of the models $B, C, D \& E$, describing loci that show some loss of methylation in one or more of the single $d c l$ mutants, but not in either the double $d c l 2 / 4$ or triple $d c l 2 / 3 / 4$ mutants. These loci are predominantly found in the $\mathrm{CpG}$ context. Model $\mathrm{F}$ is also predominantly found in CpG context methylation, and describes loci which show a gain in methylation in all $d c l$ mutants relative to the wild-type samples. Similarly, Model G represents a gain in methylation in the majority of the $d c l$ mutants over wildtype and the $d c l 2$ mutant, and is found with confidence only in the CHG methylation context. Models H, I \& J represent the canonical changes in sRNA-linked methylation 


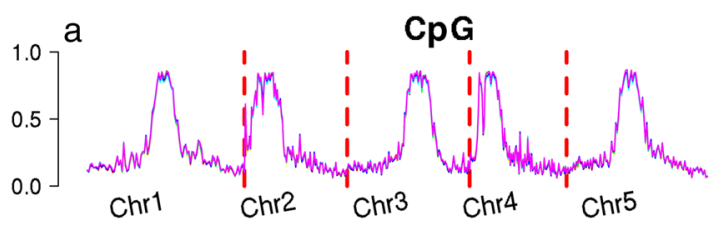

b

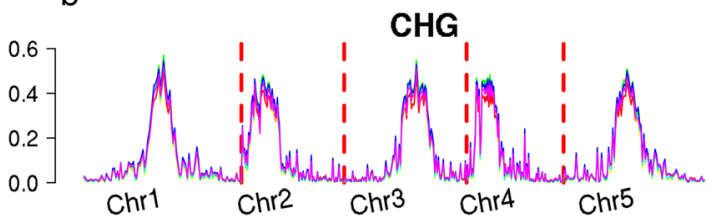

C
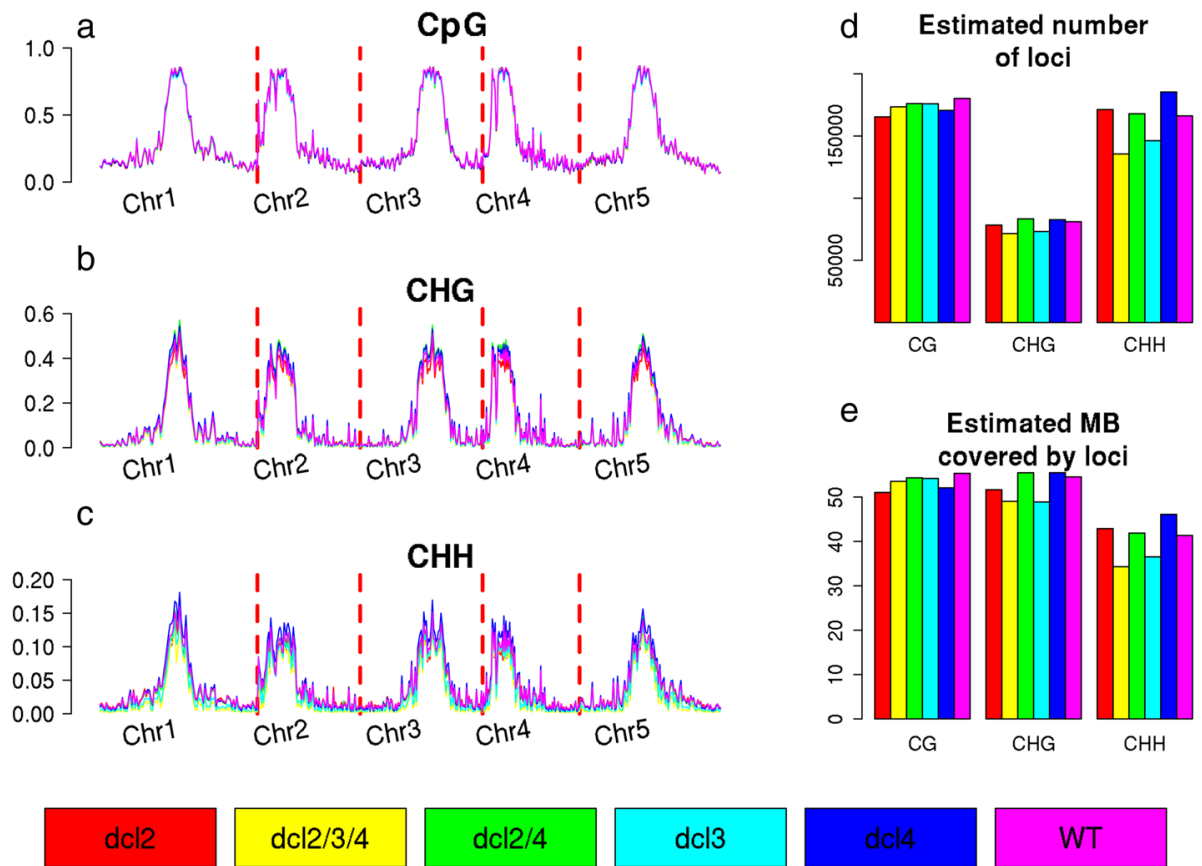

dcl3

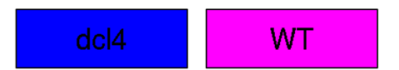

Fig. 2 Genome wide profiles of methylation for the various Dicer-like mutants, and wild-type, in $\mathrm{CpG}$ (a) $\mathrm{CHG}$ (b) and CHH (c) contexts, adjusted for non-conversion rates. The estimated number of loci identified in each condition are shown in (d), while the estimated length of the genome covered by loci are shown in (e)

[20], in which there is loss of methylation in either $d c l 3$ and $d c l 2 / 3 / 4$ relative to wild-type, with Model I somewhat exceptional in that it does not represent a loss of methylation in the $d c l 3$ mutant but only in the $d c l 2 / 3 / 4$ triple mutant. These loci are predominantly found in CHG and $\mathrm{CHH}$ contexts, conforming to the expectation that DCL3 is particularly relevant to the CHG and $\mathrm{CHH}$ methylation pathways.

The level of change in methylation varies considerably between models and contexts (Fig. 4). For example, the average loss of methylation specific to the $d c l 2$ mutant (model B) in the CpG mutant is substantial, whereas that specific to the $d c l 4$ mutant (model $\mathrm{C}$ ) is much lower (though still detectable at large numbers of loci). Gain in methylation in some or all of the $d c l$ mutants can also be substantial (model F; all contexts) or marginal (model G). For CpG context methylation, several of the more significant changes in methylation occur in loci with a short average width (Additional file 1: Figure S1), notably those in models B, E \& F, though this does not necessarily negate their biological significance [21].

Some evidence for the biological relevance of the identified classes can be acquired by examining the average methylation profiles for these loci across a range of additional mutants from the Stroud et al. (2013) [12] dataset (Additional file 1: Figure S2). In the CpG context, loci representing models $\mathrm{B}, \mathrm{C} \& \mathrm{D}$, in which methylation is lost in the $d c l 2, d c l 4$ or $d c l 2 / 4$ mutants also show a substantial average loss of methylation in the met 1 heterozygous mutant, while this effect is much reduced in the non-differential loci (model A) and those loci showing a loss of methylation in the $d c l 3$ mutant (model $\mathrm{E}$ ). This effect appears even stronger in loci showing a gain in methylation in all $d c l$ mutants over wildtype. Conversely, loci representing models $C \& D$ show a reduced loss of CpG methylation in the $d d m 1$ mutant, perhaps implying a partial independence of these loci from the chromatin remodelling methylation pathway [22].

In the CHG context, it is notable that the loci showing small gains in average methylation observed in all $d c l$ mutants except $d c l 2$ over wild-type (model G) show a similar gain in the ago 4 and nrpd1 mutants, supporting the role of the sRNA pathways in repressing methylation at these loci. Also of note is the relative independence of methylation from CMT3 in the loci representing model J, coupled with an increased dependendence on DRM1/2. This suggests a refinement of the model for redundant maintainence of CHG methylation by DRM and CMT3 proposed in Cao et al. (2003) [23] as it indicates that for some RdDM loci it is DRM that is primarily required, and that this correlates with specific patterns of differential behaviour between $d c l 3$ and $d c l 2 / 3 / 4$. In the $\mathrm{CHH}$ context, perhaps the most notable feature is the partial maintainence of methylation in the met $1 / \mathrm{cm} t 3$ mutant in the RdDM loci (models H, I \& J). Notably, the average methylation across these loci in the met1/cmt3 mutant is 


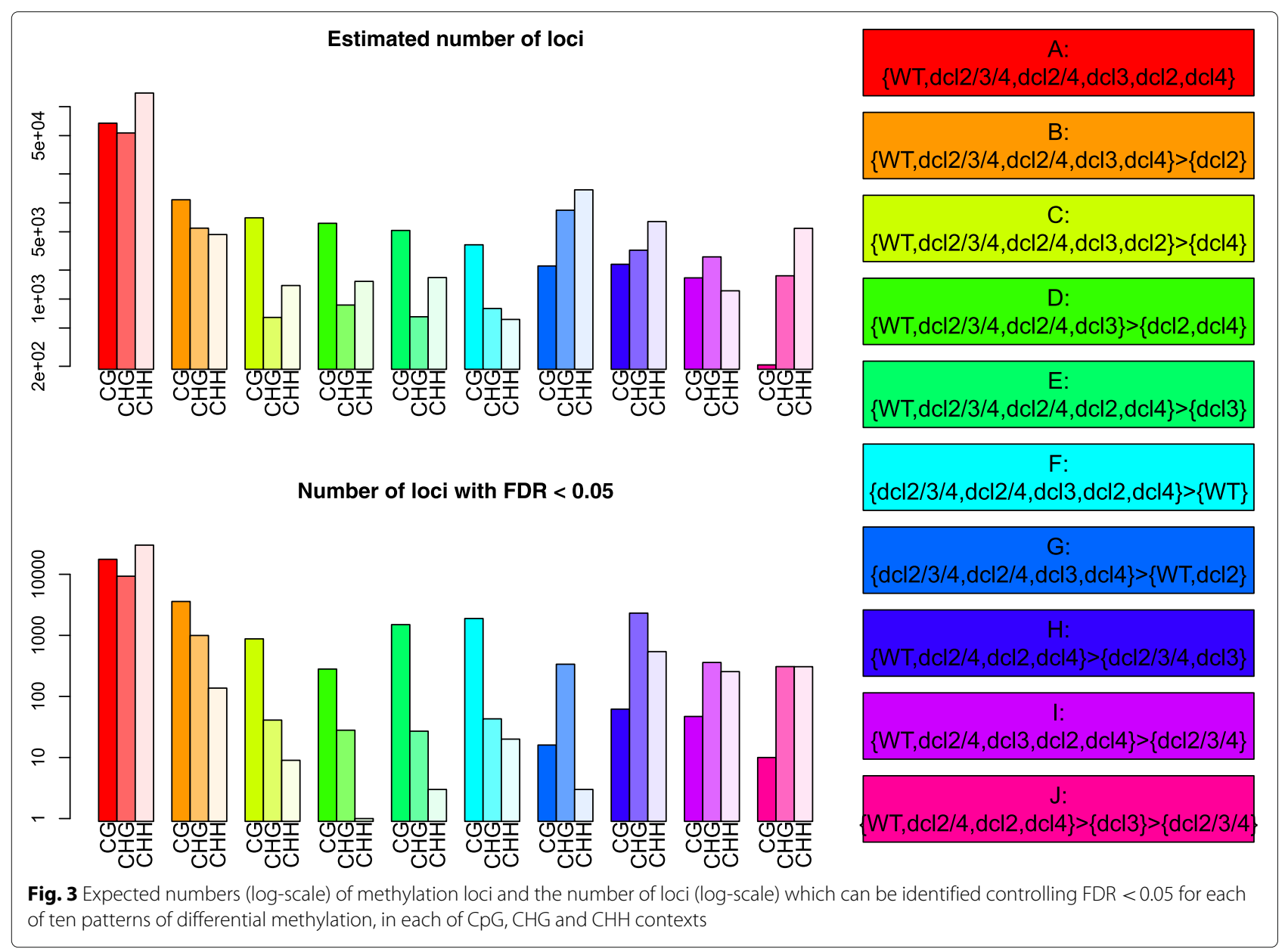

greatest in those loci affected only in $d c l 2 / 3 / 4$ and not $d c l 3$ (model I), perhaps indicating that methylation at these loci is more strongly regulated at the establishment phase by 21/22-nt sRNAs [20].

Variation is also marked in the genomic localisation of these models (Additional file 1: Figure S3). Loci representing models $B, C \& D$, in which methylation is reduced in either or both of the $d c l 2$ and $d c l 4$ mutants, but neither of the double $(d c l 2 / 4)$ or triple $(d c l 2 / 3 / 4)$ mutants are found ubiquitously across the genome in the $\mathrm{CpG}$ context but are heavily centromeric in $\mathrm{CHG}$ and $\mathrm{CHH}$ contexts. Conversely, those loci in which methylation is

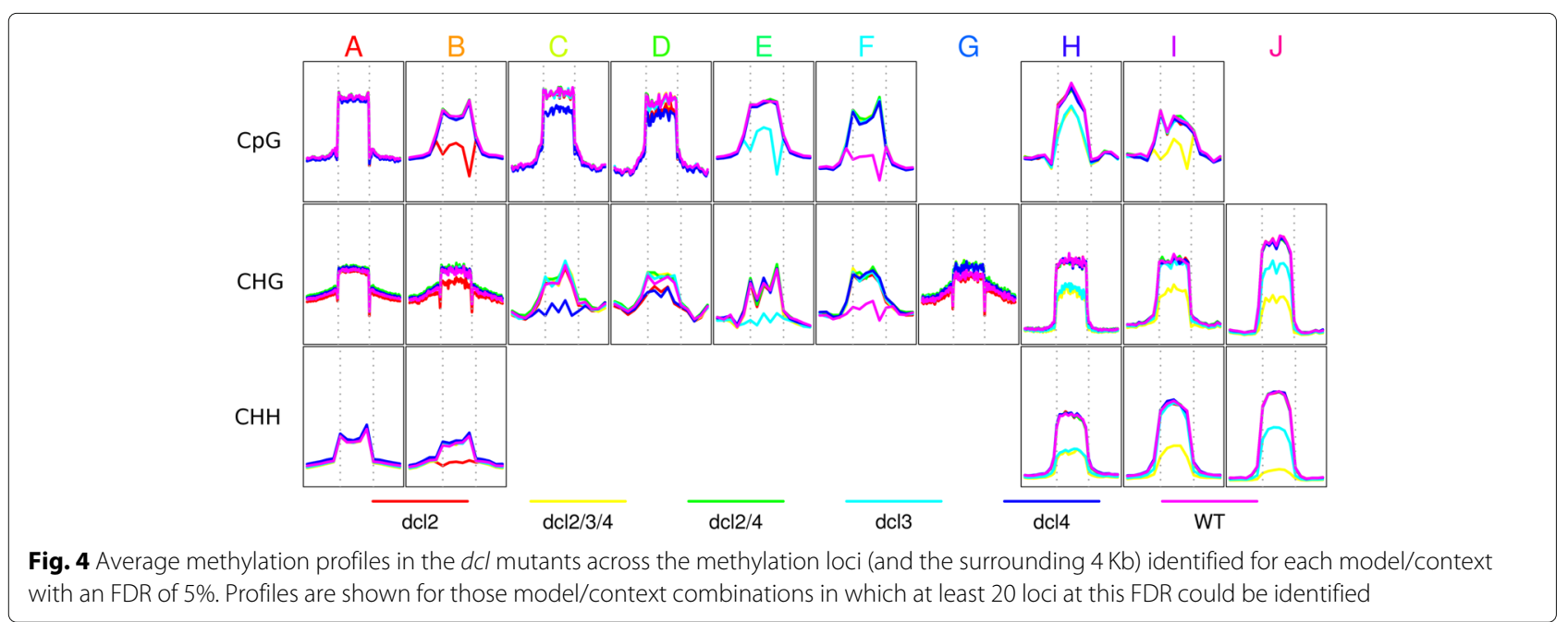


reduced only in the $d c l 3$ mutant is centromeric in the CpG context. Gains in methylation in some or all of the $d c l$ mutants appear evenly distributed across the genome in the $\mathrm{CpG}$ context but are strongly centromeric in the CHG context.

\section{Simulated data}

We next compare the performance of the approach developed here to several existing methods for detection of differential methylation using simulation data generated by WGBSSuite [11]. This tool simulates differentially methylated regions based on a complex parameter set allowing a variety of methylation types to be generated. We simulate data based on an analysis of CpG methylation in Arabidopsis (see Additional file 1 for parameter details). Using these basic parameters, we evaluate the performance of each method, varying coverage, number of replicates, the magnitude of methylation difference between replicates.

We modified the standard WGBSSuite analysis by including the effects of sample specific non-conversion rates to the data. Non-conversion rates were estimated from the wild-type and various $d c l$-mutants in the Stroud et al. (2013) [12] dataset, as above. Parameters for a beta distribution approximating the distribution of observed rates were estimated by maximum likelihood. These parameters were then used to simulate non-conversion rates for each sample in a simulation.

The simulated data are evaluated using BSmooth/bsseq [24], MethylKit [25], MethylSig [26] and MethPipe [27]. BSmooth, MethylKit, and MethylSig are implemented as in the WGBSSuite benchmarking, as is a Fisher exact test. These methods primarily rely on the detection of differential methylation at the cytosine level, and construct DMRs from the identified differentially methylated cytosines. MethPipe offers two different implementations, the first similarly based on scores constructed at each cytosine supported by a two-state hidden Markov model used to identify regions of methylation (MethPipe-1), while the second uses a beta-binomial regression on the observed data and is recommended for larger sample sets (MethPipe-2).

Performance of the methods is evaluated primarily by constructing a ranked list of DMRs based on each method's test statistic. As in WGBSSuite's benchmarking, true postives are defined as the number of truly differentially methylated cytosines within identified DMRs, while false positives are the non-differentially methylated cytosines within the identified DMRs. Figure 5 shows a comparison between the methods for data simulated using parameters intended to produce data similar to those observed in CpG methylation in plant systems. Analyses are carried out using 1,3, and 10 replicates, and with changes in the proportion of methylated cytosines between experimental groups of $0.05,0.25$ and 0.85 .
In all simulations, the segmentSeq/baySeq methods described here perform as well or better than the other methods considered as assessed by the ROC (Receiver operating characteristic) curves. The segmentSeq approach failing to account for non-conversion on average performs well, but shows greater variation and some loss of performance compared to the segmentSeqNC method which incorporates adjustments for nonconversion. This is particularly true for the experiments with few replicates; with higher numbers of replicates the effect of non-conversion will tend to average out across samples.

For large differences (a proportion shift of 0.85) in methylation all methods are able to detect differentially methylated cytosines in three and ten sample cases with almost perfect accuracy, with the exception of BSmooth and MethylKit. BSmooth shows reduced performance compared to other methods in the ten sample case and MethylKit is unable to make valid calls in any analysis. This is likely due to the design of MethylKit; it is primarily intended for the analysis of reduced representation bisulphite sequencing (RRBS) and does not appear suitable for the substantially lower coverage used in these simulations. For smaller shifts in methylation proportion the increase in performance through the segmentSeq/baySeq approach is more dramatic; this is to be expected as the increased data available to analyse methylation loci rather than individual cytosines gives greater power to detect small differences in methylation. In the analysis without replicates, the segmentSeq/baySeq approach shows substantially better performance over MethPipe and BSmooth for low and moderate differential methylation, with the MethPipe-1 analysis approaching this performance in the high differential case.

\section{Discussion}

A number of methods have previously been developed to analyse high-throughput sequencing of the methylome [7]. These are predominantly focused on the identification of differential methylation and the discovery of differentially methylated regions from grouping differentially methylated cytosines. The methods presented here adopt an alternative strategy in which first methylated and un-methylated regions are identified, and differential methylation is subsequently evaluated. Comparisons on simulated data show that the approach developed here offers substantially more power to detect small changes in methylation across a region when compared to existing methods which operate on a cytosine-by-cytosine scale, without any loss of power in the detection of large shifts in methylation. Accounting for non-conversion rates, where possible, gives a small but consistent improvement in performance, particularly when replication or the level of change in methylation is low. This is perhaps of particular 
Replicates: 1; DM: 0.05

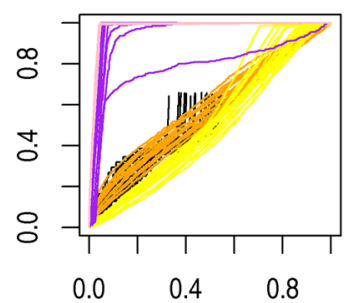

FPR

Replicates: 3; DM: 0.05

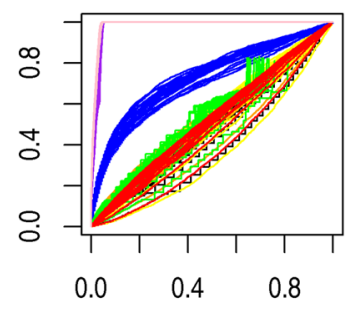

FPR

Replicates: 10; DM: 0.05

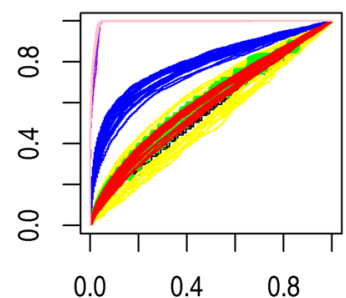

FPR

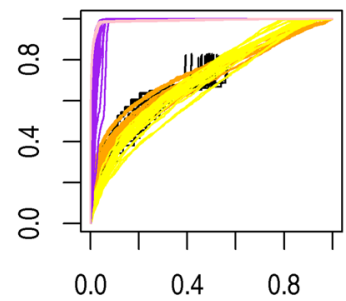

FPR
Replicates: 1; DM: 0.85

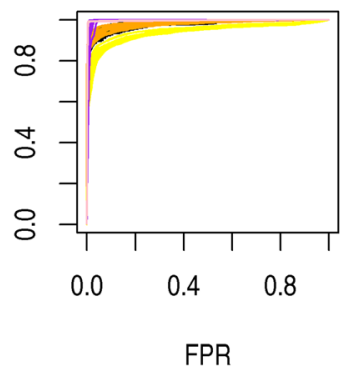

Replicates: 3; DM: 0.85

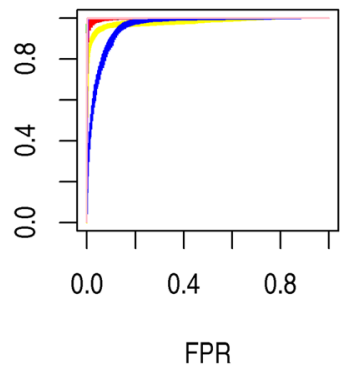

Replicates: 10; DM: 0.85

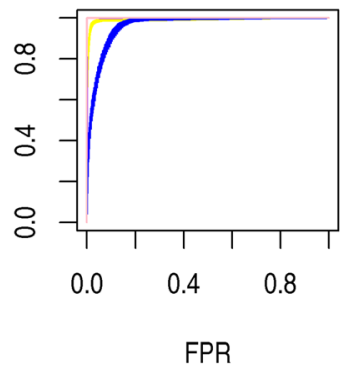

FPR

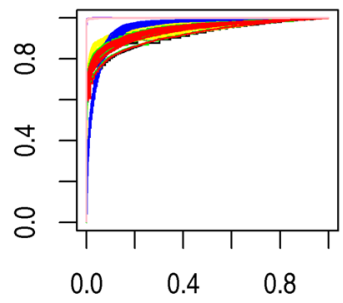

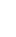

FPR

segmentSeq segmentSeq-NC

Fig. 5 ROC curves from simulated data based on WGBSSuite analyses of CpG methylation in Arabidopsis. Analyses for 1, 3 and 10 replicates are shown, and for small (0.05), moderate (0.25) and large (0.85) changes in methylation in the differentially methylated regions. Twenty simulations were carried out for each choice of replicate number/difference in methylation and curves for each simulation are shown here

importance in analysing plant methylomes, in which wildtype levels of $\mathrm{CHG}$ and $\mathrm{CHH}$ context methylation are expected to be low, and consequently loss of methylation is marked by only a small shift in the observed data.

We demonstrate our methods on a subset of the Stroud et al. (2013) [12] dataset describing the Arabidopsis methylome. A primary strength of the approach presented here is its ability to analyse complex relationships between multiple replicate groups. We demonstrate this by the simultaneous analysis of all the $d c l$ mutants, together with the wild-type samples contained in Stroud et al. (2013) [12]. Several novel patterns of methylation are identified through this analysis; most particularly a set of over a thousand CpG loci which lose methylation in the $d c l 2$ mutant but not the $d c l 2 / 4$ double mutant; at somewhat fewer loci we identify similar patterns in $\mathrm{CHG}$ and $\mathrm{CHH}$ contexts. Similarly, we identify loci which show a reduction in methylation in the $d c l 4$ mutant but not the $d c l 2 / 4$ double mutant, and loci which show a reduction in the $d c l 3$ but not the $d c l 2 / 3 / 4$ triple mutant. The mechanisms associated with these loci are not directly explicable from the data but it seems likely that there is an antagonistic relationship between the DCL-proteins at at least some of the loci, as previously noted by Bouche et al. (2006) [28]. Support for these loci as biologically meaningful is demonstrated through comparisons with 
additional mutants of the methylome regulation pathways and through analysis of the genome localisation of the discovered loci.

\section{Conclusions}

The methods described here allow for the identification of methylation loci from large sets of experimental conditions, the estimation of likelihoods for each condition that a region is truly methylated above background levels, and ultimately the detection of differential methylated regions. This approach allows downstream comparison between differentially methylated regions, non-differentially methylated regions, and nonmethylated regions. Based on comparisons on simulated data, these methods also offer a number of significant performance advantages over existing methods for detection of differential methylation, particularly in the detection of small changes in methylation levels and in experiments with low numbers of replicates. These methods also allow for the analysis of complex experimental designs, as demonstrated on a reanalysis of methylation in a set of $d c l$ mutants. This analysis demonstrates the potential utility of this method in identifying a variety of methylation loci demonstrating novel interactions between regulatory mechanisms of methylation. Though tested on methylation data derived from plant systems, there is no reason these methods should not be equally applicable to animal and human data given the conservation of $\mathrm{CpG}$ methylation between eukaryotes [29].

The methods are implemented and released within the segmentSeq [9] and bayseq [10], available on Bioconductor (www.bioconductor.org) [30]. In addition to usability and maintainence advantages, this ensures compatibility with the analyses of sRNA-seq, mRNAseq and other high-throughput data already developed in these packages. Results acquired by high-throughput sequencing of methylation can thus be readily integrated with these other -omic data, allowing the differential methylome to be incorporated into in systems level analyses.

\section{Additional file}

Additional file 1: Supplemental.pdf - supplementary materials.

Description of simulation studies and parameter details; Figure S1: Boxplot of locus widths for each model/context; Figure S2: Profiles of cytosine methylation in additional mutants for identified differentially methylated loci; Figure S3: Profiles of model abundance across the genome; Table S1: Numbers of loci associated with models of differential methylation. (PDF $3164 \mathrm{~kb})$

\section{Abbreviations}

DCL: Dicer-like (protein); DMR: Differentially methylated regions; FDR: False discovery rate; sRNA: Small RNA; RdDM: RNA-directed DNA methylation; RRBS: Reduced representation bisulphite sequencing

\section{Acknowledgments}

The author thanks Dr. Owen Rackham for his assistance in making WGBSSuite available for this study.

\section{Funding}

This work was supported by European Research Council Advanced Investigator Grant ERC-2013-AdG 340642 - TRIBE.

\section{Availability of data and materials}

The bisulfite sequencing data used in this manuscript were released to GEO by Stroud et al. [12] with accession numbers GSE39901 and GSE38286. The segment Seq and bayseq tools are availabled on Bioconductor (http:// www.bioconductor.org). WGBSSuite [11] was made available on Github (https://github.com/SystemsGeneticsSG/WGBSSuite) by Dr. Owen Rackham.

Authors' contributions

TJH is solely responsible for this study.

Ethics approval and consent to participate

Not applicable.

\section{Consent for publication}

Not applicable.

\section{Competing interests}

The author declares that he has no competing interests.

\section{Publisher's Note}

Springer Nature remains neutral with regard to jurisdictional claims in published maps and institutional affiliations.

Received: 19 May 2017 Accepted: 11 September 2017

Published online: 18 September 2017

\section{References}

1. Zhang X, Yazaki J, Sundaresan A, Cokus S, Chan SW-L, Chen H, Henderson IR, Shinn P, Pellegrini M, Jacobsen SE, Ecker JR. Genome-wide high-resolution mapping and functional analysis of DNA methylation in arabidopsis. Cell. 2006;126(6):1189-201. doi:10.1016/j.cell.2006.08.003.

2. Berdasco M, Alcázar R, García-Ortiz MV, Ballestar E, Fernández AF, Roldán-Arjona T, Tiburcio AF, Altabella T, Buisine N, Quesneville $H_{\text {, }}$ Baudry A, Lepiniec L, Alaminos M, Rodríguez R, Lloyd A, Colot V, Bender J, Canal MJ, Esteller M, Fraga MF. Promoter DNA hypermethylation and gene repression in undifferentiated Arabidopsis cells. PloS ONE. 2008;3(10):3306. doi:10.1371/journal.pone.0003306.

3. Tsukahara S, Kobayashi A, Kawabe A, Mathieu O, Miura A, Kakutani T. Bursts of retrotransposition reproduced in Arabidopsis. Nature. 2009:461(September):3-7. doi:10.1038/nature08351.

4. Clark SJ, Statham A, Stirzaker C, Molloy PL, Frommer M. DNA methylation: bisulphite modification and analysis. Nat Protoc. 2006; 1(5): 2353-64. doi:10.1038/nprot.2006.324.

5. Bock C. Analysing and interpreting DNA methylation data. Nat Rev Genet. 2012;13(10):705-19. doi:10.1038/nrg3273.

6. Hardcastle TJ. High-throughput sequencing of cytosine methylation in plant DNA. Plant Methods. 2013;9(1):16.

7. Robinson MD, Kahraman A, Law CW, Lindsay H, Nowicka M, Weber LM Zhou X. Statistical methods for detecting differentially methylated loci and regions. Front Genet. 2014;5:324. doi:10.3389/fgene.2014.00324.

8. Zhao JH, Fang YY, Duan CG, Fang RX, Ding SW, Guo HS, Bird A, Chan SW, Henderson IR, Jacobsen SE, Zhang X, Lister R, Lindroth AM, Cao X, Jacobsen SE, Matzke M, Kanno T, Daxinger L, Huettel B, Matzke AJ, Zhang H, Zhu JK, Dalakouras A, Wassenegger M, Zhao M, Leon DS, Delgadillo MO, Garcia JA, Simon-Mateo C, Dalakouras A, Dadami E, Zwiebel M, Krczal G, Wassenegger M, Wierzbicki AT, Haag JR, Pikaard CS, Law JA, Jacobsen SE, Wu L, Mao L, Qi Y, Nuthikattu S, Stroud H, Greenberg MV, Feng S, Bernatavichute YV, Jacobsen SE, Lee TF, Wang H, Hao L, Shung CY, Sunter G, Bisaro DM, Wang H, Buckley KJ, Yang X, Buchmann RC, Bisaro DM, Buchmann RC, Asad S, Wolf JN, Mohannath G, Zhang Z, Yang X, Ivanov Kl, Canizares MC, Li HW, Guo HS, Ding SW, Gonzalez I, Duan CG, Hamera S, Song X, Su L, Chen X, Fang R, Feng L, Duan CG, Guo HS, Cokus SJ, Li Y, Wang H, Mi S, Takeda A, Iwasaki S, 
Watanabe T, Utsumi M, Watanabe Y, Girard A, Hannon GJ, Sarazin A, Voinnet O, Creasey KM, Zhang X, Zhong X, Mosher RA, Schwach F, Studholme D, Baulcombe DC, Mirouze M, Mari-Ordonez A, Slotkin RK, Han BW, Wang W, Li C, Weng Z, Zamore PD, Mohn F, Handler D, Brennecke J, Siomi H, Siomi MC, Calvi BR, Gelbart WM, Dupressoir A, Heidmann T, Pasyukova E, Nuzhdin S, Li W, Flavell AJ, Ostertag EM, Lau NC, Brennecke J, Brower-Toland B, Carmell MA, Zahid K, Allen GC, Flores-Vergara MA, Krasynanski S, Kumar S, Thompson WF, Li R, Pathak RR, Lochab S, Mortazavi A, Williams BA, McCue K, Schaeffer L, Wold B, Kim KI, van de Wiel MA. Genome-wide identification of endogenous RNAdirected DNA methylation loci associated with abundant 21-nucleotide siRNAs in Arabidopsis. Sci Rep. 2016;6:36247. doi:10.1038/srep36247.

9. Hardcastle TJ, Kelly KA, Baulcombe DC. Identifying small interfering RNA loci from high-throughput sequencing data. Bioinformatics. 2012;28(4): 457-63. doi:10.1093/bioinformatics/btr687.

10. Hardcastle TJ. Generalized empirical Bayesian methods for discovery of differential data in high-throughput biology. Bioinformatics. 2016;32(2): 195-202. doi:10.1093/bioinformatics/btv569.

11. Rackham OJL, Dellaportas P, Petretto E, Bottolo L. WGBSSuite: simulating whole-genome bisulphite sequencing data and benchmarking differential DNA methylation analysis tools. Bioinformatics. 2015;31(14): 2371-3. doi:10.1093/bioinformatics/btv114.

12. Stroud H, Greenberg MC, Feng S, Bernatavichute $Y$, Jacobsen S. Comprehensive Analysis of Silencing Mutants Reveals Complex Regulation of the Arabidopsis Methylome. Cell. 2013;152(1):352-64. doi:10.1016/j.cell.2012.10.054.

13. Chapman EJ, Carrington JC. Specialization and evolution of endogenous small RNA pathways. Nat Rev Genet. 2007;8(11):884-96. doi:10.1038/nrg2179.

14. Gasciolli V, Mallory AC, Bartel DP, Vaucheret H. Partially Redundant Functions of Arabidopsis DICER-like Enzymes and a Role for DCL4 in Producing trans-Acting siRNAs. Curr Biol. 2005;15(16):1494-500. doi:10.1016/j.cub.2005.07.024.

15. Panda K, Slotkin RK. Proposed mechanism for the initiation of transposable element silencing by the RDR6-directed DNA methylation pathway. Plant Signal Behav. 2013;8(8). doi:10.4161/psb.25206.

16. Bond DM, Baulcombe DC. Epigenetic transitions leading to heritable, RNA-mediated de novo silencing in Arabidopsis thaliana. Proc Natl Acad Sci. 2015;112(3):917-22. doi:10.1073/pnas.1413053112.

17. Matzke MA, Kanno T, Matzke AJM. RNA-Directed DNA Methylation: The Evolution of a Complex Epigenetic Pathway in Flowering Plants. Annu Rev Plant Biol. 2015;66(1):243-67. doi:10.1146/annurev-arplant-043014-114633.

18. Hardcastle TJ, Kelly KA. Empirical Bayesian analysis of paired high-throughput sequencing data with a beta-binomial distribution. BMC Bioinforma. 2013;14(1):135. doi:10.1186/1471-2105-14-135.

19. Krueger F, Andrews SR. Bismark: a flexible aligner and methylation caller for Bisulfite-Seq applications. Bioinformatics. 2011;27(11):1571-2. doi:10.1093/bioinformatics/btr167.

20. Bond DM, Baulcombe DC. Small RNAs and heritable epigenetic variation in plants. Trends Cell Biol. 2014;24(2):100-7. doi:10.1016/j.tcb.2013.08.001.

21. Xu J, Pope SD, Jazirehi AR, Attema JL, Papathanasiou P, Watts JA, Zaret KS, Weissman IL, Smale ST. Pioneer factor interactions and unmethylated CpG dinucleotides mark silent tissue-specific enhancers in embryonic stem cells. Proc Natl Acad Sci U S A. 2007;104(30):12377-82. doi:10.1073/pnas.0704579104.

22. Zemach A, Kim MY, Hsieh PH, Coleman-Derr D, Eshed-Williams L, Thao K, Harmer S, Zilberman D. The Arabidopsis Nucleosome Remodeler DDM1 Allows DNA Methyltransferases to Access H1-Containing Heterochromatin. Cell. 2013;153(1):193-205. doi:10.1016/j.cell.2013.02.033.

23. Cao X, Aufsatz W, Zilberman D, Mette MF, Huang MS, Matzke M, Jacobsen SE. Role of the DRM and CMT3 methyltransferases in RNA-directed DNA methylation. Curr Biol. 2003;13(24):2212-7.

24. Hansen KD, Langmead B, Irizarry RA. BSmooth: from whole genome bisulfite sequencing reads to differentially methylated regions. Genome Biol. 2012;13(10):83. doi:10.1186/gb-2012-13-10-r83.

25. Akalin A, Kormaksson M, Li S, Garrett-Bakelman FE, Figueroa ME, Melnick A, Mason CE. methylKit: a comprehensive R package for the analysis of genome-wide DNA methylation profiles. Genome Biol. 2012;13(10):87. doi:10.1186/gb-2012-13-10-r87.

26. Park Y, Figueroa ME, Rozek LS, Sartor MA. MethylSig: a whole genome DNA methylation analysis pipeline. Bioinformatics. 2014;30(17):2414-2. doi:10.1093/bioinformatics/btu339.
27. Song Q, Decato B, Hong EE, Zhou M, Fang F, Qu J, Garvin T, Kessler M, Zhou J, Smith AD. A reference methylome database and analysis pipeline to facilitate integrative and comparative epigenomics. PloS ONE. 2013;8(12):81148. doi:10.1371/journal.pone.0081148.

28. Bouché N, Lauressergues D, Gasciolli V, Vaucheret H. An antagonistic function for Arabidopsis DCL2 in development and a new function for DCL4 in generating viral siRNAs. EMBO J. 2006;25(14):3347-56. doi:10.1038/sj.emboj.7601217.

29. Zemach A, McDaniel IE, Silva P, Zilberman D. Genome-wide evolutionary analysis of eukaryotic DNA methylation. Science. 2010;328(5980):916-9. doi:10.1126/science.1186366.

30. Gentleman RC, Carey VJ, Bates DM, Bolstad B, Dettling M, Dudoit S, Ellis B, Gautier L, Ge Y, Gentry J, Hornik K, Hothorn T, Huber W, lacus S, Irizarry R, Leisch F, Li C, Maechler M, Rossini AJ, Sawitzki G, Smith C, Smyth G, Tierney L, Yang JYH, Zhang J. Bioconductor: open software development for computational biology and bioinformatics. Genome Biol. 2004;5(10):80. doi:10.1186/gb-2004-5-10-r80.

\section{Submit your next manuscript to BioMed Central and we will help you at every step:}

- We accept pre-submission inquiries

- Our selector tool helps you to find the most relevant journal

- We provide round the clock customer support

- Convenient online submission

- Thorough peer review

- Inclusion in PubMed and all major indexing services

- Maximum visibility for your research

Submit your manuscript at www.biomedcentral.com/submit

.

\author{
(1)
}

(1) 